\title{
Kinderheilkunde im Spiegel der Kinderrechtskonvention
}

\section{Paediatrics in light of the Convention on the Rights of the Child}

() (1) $\circledast$

\author{
Autor \\ Christoph Klein \\ Institut \\ Dr. von Haunersches Kinderspital - Kinderklinik und \\ Kinderpoliklinik der LMU, München

\section{Schlüsselwörter} \\ UN-Kinderrechtskonvention, Kindeswohl, Kinderklinik
}

Key words

Convention on the Rights of the Child, best interests of the child, children's hospital

\author{
Bibliografie \\ DOI https://doi.org/10.1055/s-0043-118782 \\ Online-Publikation: 16.10.2017 \\ Gesundheitswesen 2018; 80: 191-196 \\ (c) Georg Thieme Verlag KG Stuttgart · New York \\ ISSN 0941-3790

\section{Korrespondenzadresse} \\ Prof. Christoph Klein \\ Dr. von Haunersches Kinderspital - Kinderklinik und \\ Kinderpoliklinik der LMU \\ Lindwurmstraße 4 \\ 80337 München \\ Christoph.Klein@med.uni-muenchen.de
}

\section{ZUSAMMENFASSUNG}

Die Kinderrechtskonvention der Vereinten Nationen ist nicht nur ein völkerrechtlich bindendes Dokument, das Kindern umfassende Rechte gewährt, sondern ist auch im Rang eines einfachen Gesetzes in Deutschland verbindlich. Mit Blick auf die medizinische Versorgung ist darin das „Recht auf das erreichbare Höchstmaß an Gesundheit“ sowie das Prinzip des „Vorrangs des Kindeswohls“ bei allen Entscheidungen öffentlicher oder privater Institutionen festgeschrieben. In diesem Beitrag sollen einige grundlegende Aspekte der Kinderrechtskonvention und der Monitorierung ihrer Umsetzung dargestellt sowie aktuelle Entwicklungen der Kinder- und Jugendmedizin in Deutschland im Licht der Konvention beleuchtet werden.

\section{ABSTRACT}

The United Nations Convention on the Rights of the Child not only grants comprehensive rights to children via international law, it also constitutes a legally binding framework under German federal law. With respect to health care, the convention establishes the "right of the child to the enjoyment of the highest attainable standard of health" and the principle that "the best interests of the child shall be a primary consideration" in all actions undertaken by public or private institutions. Here, some fundamental aspects of the convention and the monitoring of its implementation are summarized and current challenges in paediatric medicine in Germany are discussed in light of the convention.

\section{Einleitung}

Die UN-Kinderrechtskonvention (Convention on the Rights of the Child; (RC) wurde im Jahr 1989 verabschiedet und im Laufe der folgenden Jahre von allen Nationen der Erde - mit Ausnahme der Vereinigten Staaten von Amerika - ratifiziert. Damit haben die Vertragsstaaten deutlich gemacht, dass Kinder eigene Rechtssubjekte sind. Dreh- und Angelpunkt der CRC ist die Forderung, dass das Kindeswohl vorrangig zu berücksichtigen sei: „Bei allen Maßnahmen, die Kinder betreffen, gleichviel ob sie von öffentlichen oder privaten Einrichtungen der sozialen Fürsorge, Gerichten, Verwaltungsbehörden oder Gesetzgebungsorganen getroffen werden, ist das Wohl des Kindes ein Gesichtspunkt, der vorrangig zu berücksichtigen ist" (Art 3.1).

Alle Vertragsstaaten sind verpflichtet, die in der Konvention deklarierten Rechte in ihren jeweiligen Staatsgebieten umzusetzen und diese zu achten, zu schützen und zu gewährleisten.

Fast dreißig Jahre nach der Verabschiedung der CRC gibt es allerdings immer noch große Versäumnisse und Hindernisse im Blick auf ihre Umsetzung in nationales Recht und in der gesellschaftlichen Wirklichkeit - nicht nur in Schwellen- und Entwicklungsländern, sondern auch in Deutschland. Im Bereich der Pädagogik und des Kinderschutzes gibt es zwar eine hohe Sensibilität für die Bedeutung der CRC - doch im Blick auf das Gesundheitswesen ist die CRC und ihre Relevanz für die Medizin weitgehend noch unbekannt. Eine kritische Reflexion ist daher angezeigt. 


\section{Geltung der Kinderrechte und der Vorrang des Kindeswohls}

In Deutschland hat die CRC heute rechtsverbindliche Gesetzeskraft in Form eines einfachen Bundesgesetzes [1]. Dennoch bilden sich die Kinderrechte in der Rechtsprechung bis heute kaum ab (vgl. Ref [2]) und vielfältige Forderungen zur Verankerung der Kinderrechte im Grundgesetz (z. B. http://www.kinderrechte-ins-grundgesetz.de) konnten bisher keinen mehrheitlichen Konsens der politischen Parteien erzielen. Die Umsetzung der CRC in Deutschland wird durch die National Coalition, einen bundesweiten Zusammenschluss verschiedener Verbände und Initiativen (http://www.netzwerkkinderrechte.de/) kritisch begleitet und vorangetrieben.

Auf globaler Ebene wird die Implementierung der CRC durch eine interdisziplinäre Kommission der UN überwacht und begleitet, das „Committee on the Rights of the Child“ (CRC Kommission). Die CRC Kommission gibt konkrete Handlungsanweisungen für die Vertragsstaaten heraus („General Comments“), die allerdings noch nicht in offizieller deutscher Übersetzung vorliegen. Der Grad der Umsetzung der Kinderrechtskonvention wird durch die Staaten selbst festgestellt, die der Kommission alle 5 Jahre Bericht erstatten.

Die Kommission hält jede legislative, administrative und gerichtliche Institution dazu an, bei all ihren Entscheidungen und Handlungen dem Prinzip des Kindeswohls zu folgen. Dies gilt auch dann, wenn Kinder nur indirekt betroffen sind (Abs 12) [3]. Die Kommission bekräftigt weiterhin, dass umfassende Überprüfungen aller nationalen Gesetzgebungen und regulatorischen Aspekte der Verwaltungen erfolgen müssen, um das Befolgen der CRC sicherzustellen (Abs 18) [3]. Darüber hinaus muss sichergestellt werden, dass die Vorgaben der CRC gerichtlich einklagbar sind und im Falle des Konfliktes mit anderen gesetzlichen Regelwerken die CRC prävaliert (Abs 20) [3]. Die Verteilung finanzieller Ressourcen, die direkt oder indirekt Kinder betreffen, muss klar und transparent sein (Abs 51) [3]. Nur wenn „Kinder in Budgets sichtbar“ gemacht werden, kann beurteilt werden, ob Staaten ihrer Pflicht gemäß Artikel 4 der CRC nachkommen und „unter Ausschöpfung ihrer verfügbaren Mittel” alle angemessenen Maßnahmen zur Implementierung der CRC treffen.

Das Kindeswohl als „a primary consideration” ernst zu nehmen bedeutet, dass die Interessen der Kinder vor allen anderen Interessen zu berücksichtigen sind. Das Wohl des Kindes kann nicht durch andere Interessen aufgewogen werden, denen vermeintlich ähnliche Bedeutsamkeit zustehen könnte [3].

Dieses fundamentale Prinzip der CRC mag im Blick auf die konkreten Konsequenzen irritierend oder verstörend wirken - genau dies ist intendiert. Es geht um die Verwirklichung eines Rechtes, eines Prinzips und einer prozeduralen Regel (vgl. Ref [4]) Gleichwohl wird anerkannt, dass Artikel 3 der CRC im Konflikt mit anderen Prinzipien und Rechten stehen kann und im Einzelfall Abwägungen getroffen werden müssen (s. IV.A.4.in Ref [4]).

Vor diesem Hintergrund muss auch bedacht werden, inwieweit das öffentliche Gesundheitswesen in Deutschland die CRC im Hinblick auf schwer und lebensbedrohlich kranke Kinder zur Kenntnis nimmt und umsetzt.

\section{Das Kinderrecht auf Gesundheit (Art 24)}

Artikel 24 der CRC legt fest, dass Kinder ein Recht auf das „erreichbare Höchstmaß an Gesundheit" sowie auf Inanspruchnahme von Einrichtungen zur Behandlung von Krankheiten und zur Wiederherstellung der Gesundheit haben. Die CRC Kommission hat allgemeine Empfehlungen vorgestellt, um dem Primat der Kinderrechte im Gesundheitswesen gerecht zu werden [5]. Unabhängig vom Grad ihrer Entwicklung müssen alle Staaten unverzüglich Sorge tragen, dass die Kinderrechte mit Priorität und ohne jedwede Diskriminierung umgesetzt werden.

Kinder haben das Recht auf eine hochwertige, dem Stand der medizinischen Wissenschaft entsprechende Betreuung, welche Präventions-, Behandlungs- und Rehabilitationsmaßnahmen impliziert. Auch auf den Zugang zu einer adäquaten Palliativbetreuung wird hingewiesen. Explizit fordert die CRC Kommission, dass im Bereich der Primärversorgung die entsprechenden medizinischen Dienste in ausreichender Quantität und Qualität vorgehalten werden müssen sowie für alle Kinder annehmbar und zugänglich sein müssen. Auch wenn das Hauptaugenmerk der Kommentierung des Artikels 24 auf Aspekten der medizinischen Primärversorgung liegt, wird darauf hingewiesen, dass eine medizinische Sekundär- und Tertiärversorgung ebenfalls sichergestellt werden soll - nach Maßgabe der jeweiligen Möglichkeiten. In diesem Punkt wird der enormen Heterogenität der Gesundheitssysteme der Vertragsstaaten Rechnung getragen. Auch die CRC kann nicht darüber hinwegsehen, dass die ärztlichen Herausforderungen vom jeweiligen soziokulturellen Kontext abhängig sind und die einzelnen Staaten die Sicherung der Kindergesundheit mit anderen sozialstaatlichen Zielen (z. B. Bildung und sozialer Friede) abstimmen müssen.

Die CRC Kommission weist darauf hin, dass die Staaten Sorge tragen müssen, dass genügend ausgebildetes Personal für kranke Kinder zur Verfügung steht. Angemessene Regularien und Supervisionen, Vergütungen, Arbeitsbedingungen und Schulungen sind sicherzustellen.

Die CRC Kommission fordert, dass alle Barrieren, die Kindern einen Zugang zu medizinischen Diensten verwehren, darunter auch finanzielle, institutionelle und kulturelle Barrieren, identifiziert und eliminiert werden. In Übereinstimmung mit Artikel 24 §2 werden die Staaten aufgefordert, alle Aspekte, die für das Kinderrecht auf Gesundheit von Relevanz sind, zu identifizieren und einzufordern. Diese Maßnahmen implizieren unter anderem eine fundierte, evidenzbasierte Analyse derjenigen Umstände, die die Gesundheit von Kindern beeinflussen. Damit steckt die CRC Kommission einen weiten Rahmen: es geht nicht mehr nur um Prävention und Therapie definierter Krankheitsentitäten, es geht in einem sehr viel umfassenderen Sinn um die Sicherung der Gesundheit von Kindern und nachfolgenden Generationen.

Mit Sorge nimmt die Kommission eine zunehmende Bedeutung von Gesundheitsstörungen durch psychosoziale Faktoren wahr und fordert entsprechende präventive, edukatorische und kurative Maßnahmen. Nicht nur die Staaten, sondern auch nicht-staatliche Akteure tragen Verantwortung dafür, dass das Kinderrecht auf Gesundheit gesichert und umgesetzt wird. Die Kommission fordert 
alle nicht-staatlichen Organisationen, insbesondere die pharmazeutische und Gesundheitstechnologie-Industrie des privaten Sektors, sowie die Medien und alle Akteure im Gesundheitswesen auf, die Prinzipien der CRC zu beachten und nach ihnen zu handeln sowie sich dieser Adhärenz bei allen Partnern zu versichern.

Die Rechenschaftspflicht der Staaten ist eine zentrale Forderung, um die Implementierung des Rechts auf Gesundheit Wirklichkeit werden zu lassen. Es geht um Bekanntmachung des Rechts, entsprechende legislative Maßnahmen sowie um die Etablierung von Governance-Prinzipien. Das Kinderrecht auf Gesundheit wird nicht ohne eine Bereitstellung finanzieller Ressourcen umzusetzen sein. Staaten müssen die Verfügbarkeit, Zugänglichkeit, Annehmbarkeit und Qualität der Gesundheitsvor- und fürsorge für alle Kinder sicherstellen - ohne Diskriminierung einzelner Gruppen. Schließlich muss Sorge getragen werden, dass Verletzungen dieses Rechtsanspruchs gemeldet und geahndet werden können, anderenfalls bleibt der Rechtsanspruch Makulatur.

\section{Konkretionen im deutschen Gesundheitswesen}

Spiegelt die Realität des deutschen Gesundheitswesens die Forderungen der CRC wider? Auf den ersten Blick sieht es nicht so aus, als ob der Artikel 24 der CRC in grober Weise verletzt würde. Deutschland hat ein grosso modo sehr gut funktionierendes Gesundheitssystem. Alle Patienten haben im Prinzip Zugang zu einem dichten medizinischen Netz der Primär-, Sekundär- und Tertiärversorgung.

Eine Reihe von Untersuchungen und statistischen Erhebungen zeigt, dass die Bundesrepublik im internationalen Vergleich in einigen Parametern gute Ergebnisse vorweisen kann. Nach dem aktuellsten UNICEF Progress-Report liegt die Kindersterblichkeit in Deutschland bei $0,4 \%$ und damit im globalen Vergleich auf Rang 166 von 193 (auf Rang 193 liegen Finnland, Island und Luxemburg mit einer Kindersterblichkeit von je $0.2 \%$ ) [6].

Die Statistik der Bundesärztekammer weist 14.126 berufstätige Fachärzte/innen für Kinderheilkunde und Jugendmedizin aus, 7.104 von ihnen arbeiten im ambulanten Sektor, 5.853 im stationären Bereich (der Rest nimmt anderweitige Aufgaben wahr) (http://www.bundesaerztekammer.de/fileadmin/user_upload/ downloads/pdf-Ordner/Statistik2015/Stat15AbbTab.pdf).

Die Deutsche Gesellschaft für Kinderheilkunde und Jugendmedizin listet 358 Kinderkliniken im Bundesgebiet (http://www.dgkj. de/service/kinderkliniken/ (Stand 31.12.2016)). Rein statistisch gesehen kommt damit ein Kinderarzt auf 984 Kinder - allerdings gibt es große Unterschiede in der regionalen Verteilung fachärztlicher Kompetenz für die ca. 13,9 Millionen Kinder unter 18 Jahren (Bedarfsplanung und Realität der fachärztlichen Versorgung werden kontrovers diskutiert, vgl. z. B. Ref [7]).

Im Abschlussbericht des Nationalen Aktionsplans „Für ein kindergerechtes Deutschland” wird dem Gesundheitsszustand der meisten Kinder in Deutschland ein sehr gutes Zeugnis ausgestellt [8]. Es gibt konzertierte Maßnahmen, die dazu beitragen sollen, die Säuglings- und Kindersterblichkeit weiter zu verringern (GBA Neonatalmedizin) oder die gesundheitliche Grundversorgung zu erhalten und zu sichern (z. B. Vorsorgeuntersuchungen, Neonatal- screening, Impfungen) (Art 24 Abs 2 a,b). Krankheiten durch Mangelernährung sowie verschmutztes Trinkwasser sind in Deutschland heute sehr selten (Art 24 Abs 2c). Mütter und Väter genießen vor und nach der Geburt im internationalen Vergleich einen hohen Grad an sozialem Schutz (Art 24 Abs 2d), auf vielen Kanälen werden Informationen über Gesundheit und Ernährung, zu Hygiene und Umweltschutz verbreitet (Art 24 Abs 2e). Auch Maßnahmen zum weiteren Ausbau der Gesundheitsvorsorge, der Eltern- und Familienplanung sind in Deutschland nachweisbar (z. B. Aktionen der Krankenkassen, Sozialverbände etc).

Der aktuelle Bericht der Bundesregierung zum Stand der Umsetzung der CRC deklariert, dass die Förderung eines gesunden Lebens und gesunder Umweltbedingungen Schwerpunkte der deutschen Politik sind [9].

Auf den zweiten Blick allerdings werden durchaus Probleme offenbar, die Sorge bereiten müssen.

So weist die National Coalition darauf hin, dass „nicht mehr die akuten körperlichen Krankheiten, sondern die chronischen, psychischen und psychosomatischen Krankheiten sowie ungesunde Lebensstile Anlass zur Sorge geben “ [10]. Explizit genannt werden die frühen Eltern-Kind-Bindungs- und Regulationsstörungen, umweltmitbedingte Erkrankungen wie z. B. Allergien, Bewegungsmangel und Übergewicht, emotionale Entwicklungs- und Verhaltensprobleme sowie frühe Anfälligkeit für Alkohol, Nikotin und andere Drogen [10]. Diese Veränderung des Krankheitsspektrums wird auch als „neue Morbidität“ bezeichnet [11, 12]. Dabei bereitet insbesondere der Zusammenhang zwischen dem Gesundheitszustand bzw. dem Krankheitsrisiko von Kindern und soziokulturellen und -ökonomischen Determinanten Sorge, wie auch der Bericht der Bundesregierung „Perspektiven für ein kindergerechtes Deutschland“ konstatiert [8]. Hier müssen Gesellschaft und Politik ihrer Verantwortung gerecht werden und im Sinne der CRC die Rahmenbedingungen für Kinder und ihre Gesundheit verbessern.

Andererseits sind auch Kinder mit Erkrankungen, die nicht in erster Linie mit psychischen und soziokulturellen Determinanten assoziiert sind, im Gesundheitswesen unzureichend berücksichtigt. Hier ist insbesondere auf das Schicksal von Kindern hinzuweisen, die an schweren und lebensbedrohlichen Erkrankungen leiden. Da sie in der systemischen Gesamtschau aufgrund ihrer Seltenheit marginalisiert werden, wird ihr Schicksal meist vernachlässigt [10]. Gleichwohl gilt es, ihnen zu ihren in der CRC verbrieften Rechten zu verhelfen.

An folgenden Beispielen soll aufgezeigt werden, in welcher Weise die Vorgaben der CRC im deutschen Gesundheitswesen (noch) nicht auf adäquate Weise umgesetzt werden.

\section{Transparenz und Monitorierung der Implementierung der Kinderrechte im deutschen Gesundheitswesen}

Die National Coalition monitoriert mit kritischem Blick die Fortschritte bei der Implementierung der CRC. Allerdings scheint das Kinderrecht auf Gesundheit (Art 24) im Gegensatz zu den pädagogischen, juristischen und soziologischen Aspekten eher von nachrangiger Bedeutung zu sein (vgl. http://www.netzwerkkinderrechte.de/themen/gesundheit-art-24.html) und eine um- 
fassende Analyse zur Situation von Kindern im deutschen Gesundheitswesen steht aus.

Laut statistischem Bundesamt entfielen im Jahr 2008 von den insgesamt 254,3 Mrd Euro Krankheitskosten 15,2 Mrd Euro für die medizinische Betreuung von Kindern unter 15 Jahren [13]. Um der Forderung der CRC Kommission, Kinder in Budgets sichtbar zu machen, gerecht zu werden, müssten diese Daten in höherer Granularität dargestellt werden. Hier sollten Verbände (z. B. Verband der Universitätsklinika, Spitzenverbände der gesetzlichen und privaten Krankenversicherungen) angehalten werden entsprechende Darstellungen zu erarbeiten.

Universitäre Kinderkliniken und viele andere Abteilungen für Kinderheilkunde haben heute in Deutschland keine eigene Deutungshoheit mehr, sie sind in der Regel gemeinsam mit allen Kliniken der Erwachsenenmedizin eingegliedert in die Verfasstheit großer Klinika. Vor diesem Hintergrund ist weder die Ressourcenallokation für kranke Kinder ausreichend transparent dargestellt, noch werden Kindern partizipative Rechte in den „Governance-Strukturen" eingeräumt.

Nicht nur in budgetärer Hinsicht, auch in vielen anderen Aspekten wäre es wichtig, die Umsetzung der CRC im Gesundheitswesen zu monitorieren. Sind die Kinderkrankenhäuser so ausgestattet, dass kranke Kinder ihr Recht auf Spiel umsetzen können? Sind die Vorgaben der Krankenhausarchitektur im Einklang mit der CRC? Wird das Recht kranker Kinder geachtet, nicht von ihren Eltern getrennt zu werden?

Diese und viele weitere Fragen müssten auf der Basis von umfassenden Analysen (insbesondere unter Beteiligung von Kindern und Jugendlichen) beantwortet werden, damit konkrete Forderungen an Politik und Gesellschaft abgeleitet werden können. Dabei müssen auch die Adressaten für Beschwerden klar definiert werden, um die Einklagbarkeit der Kinderrechte zu gewährleisten. Ein neuer Blick auf das deutsche Gesundheitswesen ist dringend nötig - als Voraussetzung für eine Gesundheitspolitik, die die gesetzlichen Vorgaben der CRC einhält.

\section{Finanzierung der Kinderkliniken im DRG-System und ethische Fragen der Ressourcenallokation}

Seit dem Jahr 2003 erfolgt die Vergütung der stationären Behandlungen in Deutschland im Rahmen eines „durchgängigen, leistungsorientierten und pauschalierenden Vergütungssystems” ( $\S$ 17b Abs 1 Satz 1 KHG; DRG-System). Im Zuge des so geschaffenen wirtschaftlichen Konkurrenzgefüges sank die Zahl der Krankenhäuser. Die Versorgungsstrukturen für kranke Kinder waren überproportional betroffen. Die Gesellschaft der Kinderkrankenhäuser und Kinderabteilungen in Deutschland diagnostizierte einen dramatischen Rückgang der Kinderabteilungen von 440 im Jahr 1991 auf 364 im Jahr 2013. Vier von zehn Betten in der stationären Kinderund Jugendmedizin wurden eliminiert (von 31.707 in Jahr 1991 auf 19.199 Betten im Jahr 2012) [14]. An vielen Stellen wird die Betreuung kranker Kinder nicht mehr durch speziell ausgebildete Kinderkrankenschwestern, Kinderchirurgen und Kinderärzte gewährleistet, sondern durch Personal, welches in erster Linie erwachsene Patienten betreut [10].

Diese Entwicklung hat Auswirkungen auf die Versorgungsqualität kranker Kinder.
Das Ziel einer ganzheitlichen klinischen Betreuung, die das kranke Kind ins Zentrum stellt und nicht nur die biologischen, sondern auch die psychischen und sozialen Determinanten von Krankheit und Gesundheit in den Blick nimmt, entspricht dem Geist der CRC, ist aber nur sehr schwer in Einklang zu bringen mit dem auf Optimierung von Effizienzen ausgerichteten DRG-System. Ein Beispiel: Bei erwachsenen Patienten ist die bildgebende Diagnostik mittels Kernspintomographie in monetärer Hinsicht lukrativ, bei Kindern aber führt sie oft zu finanziellen Verlusten. Kinder durchbrechen die engen zeitlichen Taktungen und benötigen oft Sedierungen oder Narkosen für die Untersuchungen. Dies ist wenig kompatibel mit einem auf Effizienz und Profitabilität ausgerichteten Patientendurchfluss. Folglich werden die Zeitfenster für die Untersuchung von Kindern minimiert, was lange Wartezeiten nach sich zieht. Ähnliche Probleme ergeben sich in vielen anderen klinischen Bereichen, denn das DRG-System vernachlässigt systematisch all jene Dimensionen der Heilkunst, die sich nicht leicht in Maß und Zahl abbilden lassen. Innerhalb der Pädiatrie hat das System gefährliche strukturelle Ungleichgewichte befördert. Subdisziplinen, in denen Normierungen und Standardisierungen leichter möglich sind, wie z. B. die Neonatologie, sind „Gewinner”, die von Krankenhausträgern strukturell gefördert werden. Verlierer sind viele andere Disziplinen, von der pädiatrischen Stoffwechselmedizin bis zur Immunologie, die den Krankenhausträgern Verluste bringen und somit auf ein Mindestmaß reduziert oder gar ganz geschlossen werden. Diese Entwicklung kritisiert der Deutsche Ethikrat in seiner Stellungnahme „Patientenwohl als ethischer Maßstab für das Krankenhaus” als „höchst problematisch“ [15].

Heute gilt es, im Sinne der CRC, einer weiteren systematischen Benachteiligung kranker Kinder Einhalt zu gebieten. Dabei sollten direkte systemische Konkurrenzsituation sowohl innerhalb der Kinderheilkunde als auch zwischen der Kinderheilkunde und den Disziplinen der Erwachsenenmedizin minimiert werden.

Der Deutsche Ethikrat legt in seiner Stellungnahme dar, dass die Konkurrenzsituation umgangen werden könnte, „wenn die Kinderund Jugendmedizin komplett von dem Fallgruppen-Vergütungssystem der Erwachsenenmedizin entkoppelt würde und entweder ein kinderspezifisches eigenes DRG-System etabliert oder für die stationäre Kinder- und Jugendmedizin ganz andere Abrechnungsmodi, etwa tagesgleiche Pflegesätze, eingeführt würden“ [15].

\section{Partizipation von Kindern und Jugendlichen in den Governance Strukturen der Kliniken für Kinderheil- kunde und Jugendmedizin}

Die CRC erkennt Kinder erstmals als eigene Rechtssubjekte mit spezifischen Beteiligungsrechten an. Die Realität in den Strukturen des deutschen Gesundheitswesens wird dem allerdings kaum gerecht. In Ländern des angelsächsischen Sprachraums ist es heute eine Selbstverständlichkeit, nicht nur die Meinung erwachsener Patienten und der Eltern, sondern vielmehr die Meinung von Kindern und Jugendlichen selbst im Rahmen von regelmäßigen Befragungen zur Qualität der medizinischen und psychosozialen Betreuung einzuholen und ernst zu nehmen. Auch bei Fragen der Krankenhausarchitektur, der Ausstattung von Kliniken sollten Kinder und Jugendliche einbezogen werden. In Australien gewähren Kinderkrankenhäuser Kindern und Jugendlichen eine Einsicht und ein 
Mitspracherecht in Fragen der „Hospital Governance“ bis hin zu Fragen der Finanzen - mit guten Erfahrungen. Die Berücksichtigung der Stimme von Kindern und Jugendlichen, die Etablierung von „Governance-Strukturen“, welche partizipative Elemente integrieren, sind unerfüllte Desiderate im deutschen Gesundheitswesen.

\section{Versorgung von Kindern mit seltenen Erkrankungen}

Erkrankungen, die weniger als einen Menschen von 2.000 betreffen, gelten in Europa als seltene Krankheiten. Ca. 8.000 dieser Erkrankungen sind heute bekannt, zumeist liegt ihnen ein Webfehler im Genom zugrunde. Die medizinische Versorgung von Kindern mit seltenen Erkrankungen stellt das Gesundheitssystem vor große Herausforderungen.

Die betroffenen Kinder und ihre Familien leiden in vieler Hinsicht: oft dauert es viele Jahre, bis eine korrekte Diagnose gestellt werden kann und für die meisten seltenen Erkrankungen gibt es bis heute keine kurativen Therapieoptionen. Weder die öffentliche noch die privatwirtschaftlich finanzierte Wissenschaft widmet sich in ausreichendem Maße der Erforschung dieser seltenen Erkrankungen.

Kinder mit seltenen Erkrankungen sind nicht im Fokus der Gesundheitssysteme. Sie sind die „Waisen der Medizin”. Ihre interdisziplinäre und multiprofessionelle Betreuung erfordert eine große Zahl an Spezialisten, die nur in großen Zentren anzutreffen ist. Aufgrund der geringen Fallzahl gibt es selten eine eigene, auf soliden Berechnungen beruhende und adäquat kalkulierte Fallpauschale. Dabei leiden etwa ein Drittel aller Patienten, die in universitären tertiären Zentren betreut werden, an einer seltenen Erkrankung. „Die Fehlwahrnehmung, es handele sich um marginalisierbare Einzelphänomene, hat mit dazu beigetragen, dass es dem geltenden Gesundheits(sozial)recht - als Ensemble von Normen und Interpretationsdiskursen - bislang nicht gelungen ist, sich in ein konsistentes Verhältnis zu seltenen Erkrankungen zu bringen“, so definiert Stefan Rixen die schwierige sozialrechtliche Situation der Patienten mit seltenen Erkrankungen [16]. Ein Beispiel: Kinder mit der seltenen Ahornsiruperkrankung, einer Störung des Aminosäuremetabolismus, müssen lebenslang eine Spezialdiät zu sich nehmen, die keine verzweigtkettigen Aminosäuren enthält. Für die hohen Kosten müssen die Familien selbst aufkommen. Nach Interpretation der Sozialversicherungsträger handelt es sich ja nicht um eine medikamentöse Therapie (diese würde bezahlt werden), sondern vielmehr „nur“ um diätetische Maßnahmen, die vom Patienten selbst zu finanzieren sind. Dieses Beispiel illustriert die Fallstricke eines Systems. Immer wieder treten Situationen auf, die ganz offensichtlich kaum mit den Grundsätzen der CRC vereinbar sind. Der „Schattenbericht“ der National Coalition kritisiert: „50\% der von einer seltenen chronischen Krankheit eines Kindes betroffenen Familien fühlen sich schlecht informiert, $70 \%$ der betroffenen Familien fühlen sich allein gelassen “ [10]. In jüngster Vergangenheit gibt es politische Bestrebungen, im Rahmen des 2013 gegründeten Nationalen Aktionsbündnisses für Menschen mit Seltenen Er- krankungen (NAMSE) die Situation der Patienten mit seltenen Erkrankungen zu verbessern. Experten zweifeln allerdings, ob sich die Situation von Kindern mit seltenen Erkrankungen tatsächlich verbessert, wenn nicht, wie oben dargestellt, manche zugrundeliegenden Prinzipien aktualisiert werden.

\section{Weiterbildung in den pädiatrischen Subdisziplinen}

Mit dem Fortschritt einer wissenschaftlichen Medizin hat die Pädiatrie einen hohen Grad an Differenzierung in einzelne Subdisziplinen erfahren. Die Facharztbezeichnung „Kinderheilkunde und Jugendmedizin” genügt zwar als Qualifikation für eine Grundversorgung, die ärztliche Sorge um Kinder mit schweren und seltenen Erkrankungen allerdings muss das zunehmende Wissen der organbezogenen Spezialdisziplinen berücksichtigen.

Im deutschen Gesundheitswesen ist derzeit jedoch nicht nur die „Vorhaltung” pädiatrischer Subdisziplinen gefährdet, auch die Ausbildung von hoch-spezialisierten Kinderärzten/-innen bricht als Konsequenz des Primats der Ökonomie weg. Es gibt schlichtweg keine Abbildung der Ausbildungskosten im DRG-Vergütungssystem. Die finanziellen Zuwendungen der Bundesländer zur Finanzierung von Forschung und Lehre an den Universitäten werden für die Ausbildung von Studierenden oder zur Deckung der Defizite in der ambulanten und stationären Krankenversorgung, nicht für die Weiterbildung in den pädiatrischen Subdisziplinen eingesetzt. Andere Länder rufen kompetitive Wettbewerbe aus, damit sich akademische Kinderkliniken um entsprechende finanzielle Unterstützung bewerben können (z. B. können sich in den USA tertiäre Zentren um NIH Institutional Training Grants bewerben). In Deutschland fällt das aus. Es sind die Kinder zukünftiger Generationen, die unter dieser kurzsichtigen Gesundheitspolitik leiden werden.

Unter der Maßgabe der CRC, der Sorge um Belange von Kindern einen Vorrang einzuräumen, ist dieses aktuelle Defizit des deutschen Gesundheitswesens scharf zu kritisieren. Wenn „Kinder unsere Zukunft" sind, so sollten wir auch hier und heute Sorge tragen, dass auch in Zukunft in Deutschland eine kompetente medizinische Betreuung kranker Kinder auf hohem Niveau stattfinden kann. Dies kann nur gewährleistet werden, wenn die entsprechende Fachkompetenz auch in Ausbildungsprogrammen weitergetragen werden kann.

Alle vier beispielhaften Konkretisierungen verdeutlichen, dass die Umsetzung der Kinderrechte im deutschen Gesundheitswesen längst nicht am Ende ist. Eine umfassende und detaillierte Analyse der Ist-Situation im Blick auf die Kinderrechte, die aktive Beteiligung von Kindern und eine kritische Re-Lecture wie dringliche Adaptation der Vergütungsstrukturen im Gesundheitswesen, eine ehrliche Sorge um Kinder mit seltenen Erkrankungen und eine nachhaltige Sicherung der Ausbildungsstrukturen sind wichtige Elemente, die dazu beitragen können, dass auch in Deutschland die CRC auf umfänglichere Weise umgesetzt und implementiert wird. 


\section{FAZIT}

Derzeit steht ein alle Strukturen des Medizinsystems durchdringendes Effektivitäts-Ideologem oft im Kontrast zu den Bedürfnissen kranker Kinder. Die Respektierung der Kinderrechte, eine Monitorierung ihrer Umsetzung im Gesundheitswesen, eine Anerkennung der partizipativen Rechte kranker Kinder in Fragen der Governance von Krankenhäusern sowie die Einrichtung von Beschwerdestellen und nicht zuletzt eine aktive Beteiligung der Instanzen des Verwaltungs- und Sozialrechts sind wichtige Aspekte, damit alle relevanten Akteure im Gesundheitswesen ihr Tun an der Würde des Kindes orientieren und gemäß der Kinderrechtskonvention dem Wohl des Kindes Vorrang vor anderen Interessen einräumen. Eine Kenntnisnahme der CRC, ihre Beachtung und eine konkrete Umsetzung gemäß den oben dargestellten Forderungen ist für das deutsche Gesundheitswesen ein dringliches Desiderat - im Interesse kranker Kinder und ihrer Familien!

\section{Danksagung}

Der Autor dankt Professor Dr. G. Marckmann, Prof. Dr. H. Tremmel und Janina Klein für kritische Kommentare sowie Frau Dr. Henrike Klinker für ihre Hilfe bei der Manuskripterstellung. Die Arbeit wurde teilweise durch Mittel der Deutschen Forschungsgemeinschaft (Gottfried-Wilhelm-Leibniz-Programm) gefördert.

Interessenkonflikt

Der Autor erklärt, dass kein Interessenkonflikt besteht.

\section{Literatur}

[1] Cremer H. Die UN Kinderrechtskonvention - Geltung und Anwendbarkeit in Deutschland nach der Rücknahme der Vorbehalte. Berlin: Deutsches Institut für Menschenrechte; 2012

[2] Eichholz R. Der Vorrang des Kindeswohls. Die Bedeutung von Art 3 Abs 1 der UN-Kinderrechtskonvention für die deutsche Rechtssprechung. National Coalition in Deutschland - Netzwerk zur Umsetzung der UN Kinderrechtskonvention (Hrsg.); 2015
[3] Committee on the rights of the child. Convention on the rights of the child - General Comment No. 5 (2003) - General measures of implementation of the Convention on the Rights of the Child (arts. 4, 42 and 44, para. 6). CRC/CC/2003/5. United Nations; 2003

[4] Committee on the rights of the child. Convention on the rights of the child - General comment No. 14 (2013) On the right of the child to have his or her best interests taken as a primary consideration (art. 3, para. 1). CRC/C/GC/14. United Nations; 2013

[5] Committee on the rights of the child. Convention on the rights of the child - General comment No. 15 (2013) On the right of the child to the enjoyment of the highest attainable standard of health (art. 24). CRC/C/GC/15. United Nations; 2013

[6] UNICEF. Committing to Child Survival: A Promise Renewed. Progress Report 2015. 2015: 24

[7] Bertelsmann Stiftung (Hrsg.). Spotlight Gesundheit 03/2015. 2015

[8] Bundesministerium für Familie, Senioren, Frauen und Jugend (Hrsg.). Perspektiven für ein kindergerechtes Deutschland. 2010; 60ff

[9] Bundesministerium für Familie, Senioren, Frauen und Jugend (Hrsg.). Dritter und Vierter Staatenbericht der Bundesrepublik Deutschland zu dem Übereinkommen der Vereinten Nationen zu den Rechten der Kinder. 2010; 61ff

[10] National Coalition für die Umsetzung der UN-Kinderrechtskonvention in Deutschland (Hrsg.). Ergänzender Bericht zum Dritt- und Viertbericht der Bundesregierung an die Vereinten Nationen gemäß Art 44 Abs 1 Buchstabe $b$ des Übereinkommens für die Rechte der Kinder.Berlin: Eigenverlag Arbeitsgemeinschaft für Kinder- und Jugendhilfe 2010;24f

[11] Kurth BM, Schaffrath Rosario A. Die Verbreitung von Übergewicht und Adipositas bei Kindern und Jugendlichen in Deutschland. Bundesgesundheitbl 2007; 50: 736

[12] Hölling H, Schlack R, Petermann F et al. Psychische Auffälligkeiten und psychosoziale Beeinträchtigungen bei Kindern und Jugendlichen im Alter von 3 bis 17 Jahren in Deutschland - Prävalenz und zeitliche Trends zu 2 Erhebungszeitpunkten (2003-2006 und 2009-2012). Bundesgesundheitsbl 2014; 57: 807

[13] Statistisches Bundesamt. Gesundheit - Krankheitskosten - 2002, 2004, 2006 und 2008. Fachserie 12 Reihe 7.2. Wiesbaden 2010

[14] Deutsche Gesellschaft für Kinder- und Jugendmedizin e.V./Gesellschaft der Kinderkrankenhäuser und Kinderabteilungen in Deutschland e.V. Zahlen und Daten zur Finanzierung von Kinderkliniken und -abteilungen in Deutschland. 2014

[15] Deutscher Ethikrat. Patientenwohl als ethischer Maßstab für das Krankenhaus - Stellungnahme. Berlin 2016; $97 \mathrm{f}$

[16] Rixen S. Seltene Erkrankungen als Problem des Gesundheitssozialrechts. Z. Evid. Fortbild. Qual. Gesundh. Wesen 2008; 102: 31-36 